Cahiers de philosophie de l'université de

\title{
Bibliographie en français sur Vladimir Soloviev (1855-1900)
}

\section{Michel Niqueux}

\section{Q OpenEdition}

1 Journals

Édition électronique

URL : https://journals.openedition.org/cpuc/1046

DOI : 10.4000/cpuc.1046

ISSN : 2677-6529

Éditeur

Presses universitaires de Caen

\section{Édition imprimée}

Date de publication : 31 octobre 2011

Pagination : 205-209

ISBN : 978-2-84133-385-1

ISSN : 1282-6545

\section{Référence électronique}

Michel Niqueux, «Bibliographie en français sur Vladimir Soloviev (1855-1900) », Cahiers de philosophie de l'université de Caen [En ligne], 48 | 2011, mis en ligne le 01 septembre 2020, consulté le 02 février 2023. URL : http://journals.openedition.org/cpuc/1046; DOI : https://doi.org/10.4000/cpuc.1046

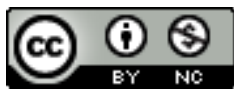

Creative Commons - Attribution - Pas d'Utilisation Commerciale 4.0 International - CC BY-NC 4.0 https://creativecommons.org/licenses/by-nc/4.0/ 


\section{Bibliographie en français sur Vladimir Soloviev ${ }^{1}$ (1855-1900)}

\section{Cuvres}

(ordre alphabétique des titres en français)

Crise de la philosophie occidentale [1874], Paris, Aubier-Montaigne (Bibliothèque philosophique), 1947 (trad. et introduction de Maxime Herman), 384 p.

Le développement dogmatique de l'Église [1885], Paris, Desclée, 1991 (trad. et présentation de François Rouleau et Roger Tandonnet), 209 p.

Les fondements spirituels de la vie [1884], Lettre préface de S. Exc. M ${ }^{\text {gr }}$ Michel d'Herbigny, Paris, Beauchesne et Bruxelles, Éditions de la cité chrétienne, 1932 (trad. par le R. P. Georges Tzebricow), 243 p.

- Paris, Casterman, 1948, 195 p.

La grande controverse et la politique chrétienne (Orient-Occident) [1883], Paris, Aubier-Montaigne (Philosophie de l'esprit), 1953 (trad. par T. D. M.), 232 p.

Histoire et avenir de la théocratie [1887], Paris, Cujas, 2008 (introduction de François Rouleau; trad. par Mireille Chmelewsky, Antoine Elens, François Rouleau, Roger Tandonnet), $286 \mathrm{p}$.

L'idée russe, Paris, Didier - Perrin, 1888, 49 p. [repris dans La Sophia et les autres écrits français].

Le judaïsme et la question chrétienne [1884], Paris, Desclée, 1992 (préface d'Alain Besançon), $192 \mathrm{p}$.

La justification du bien: essai de philosophie morale [1897], Paris, Aubier-Montaigne (Philosophie de l'esprit), 1939 (trad. par T. D. M.), 510 p.; rééd. 1997.

1. Autres graphies: Soloviov, Solovieff, Solowiew, Solov'jev, Solov'ev. 
Leçons sur la divino-humanité [Recueil de conférences données à Saint-Pétersbourg en 1877-1878], Paris, Cerf, 1991 (préface de François Rouleau ; trad. par Bernard Marchadier), $180 \mathrm{p}$.

Mahomet, Genève, Ad Solem, 2001 (trad. et présentation de Bernard Marchadier), $148 \mathrm{p}$.

La question pénale au point de vue éthique [1897], Revue internationale de sociologie, t. V, 1897, p. 514-538 (trad. par Mali Krogius).

La Russie et l'Église universelle [1889], Paris, A. Savine, 1889, 336 p.

— Lausanne, l'Âge d'homme, 1978.

— Paris, F.-X. de Guibert, 2008 (préface de Patrick de Laubier).

"Saint Vladimir et l'État chrétien », L'Univers, 4 et 11 août 1888 (http://gallica.bnf. fr/ark:/12148/bpt6k706348d).

Le sens de l'amour: essais de philosophie esthétique [1894], Paris, Aubier, 1946 (trad. par T. D. M.), 144 p.

- Paris, OEIL, 1985 (introduction de François Rouleau; trad. par Bernard Marchadier), 270 p. [contient aussi «Le drame de la vie de Platon», «La Beauté dans la nature», «Le sens général de l’art», «Un premier pas vers une esthétique positive»].

La Sophia et les autres écrits français, édité et présenté par François Rouleau, Lausanne, l'Âge d'homme, 1981, 368 p.

Trois entretiens sur la guerre, la morale et la religion [1899], Paris, Plon-Nourrit, 1916 (traduits du russe et présentés par Eugène Tavernier), $217 \mathrm{p}$.

— Paris, OEIL, 1984 (introduction de François Rouleau ; trad. et notes de Bernard Marchadier), $233 \mathrm{p}$.

- Genève, Ad Solem, 2005 (trad. et introduction de Bernard Marchadier), 188 p.

Trois rencontres et autres poèmes, Marseille, Harpo \&, 2004 (trad. par Christian Mouze), $40 \mathrm{p}$.

Patrimoine littéraire européen. Mondialisation de l'Europe. 1885-1922, anthologie en langue française sous la direction de Jean-Claude Polet, Bruxelles, De Boeck université, 200o, vol. 12, «Soloviev» (par F. Lhoest, A. Nicolaïev) [présentation et extraits de La justification du bien; Trois entretiens sur la guerre, la morale et la religion; «Le drame de la vie de Platon»], p. 132-144. 


\section{Études}

BALTHASAR Hans Urs von, La gloire et la croix. Les aspects esthétiques de la révélation, II, Styles. ${ }^{* *}$ De Jean de la Croix à Péguy, Paris, Aubier, 1972, p. 167-230.

Barabanov Eugène, «L'Église et le monde dans la pensée de V. Soloviev. Un débat oublié», Messager orthodoxe, 17, 1977, p. 3-53.

Berdiaev Nicolas, «Le problème de l'Orient et de l'Occident chrétiens dans la conscience religieuse de Vladimir Soloviev», Contacts, vol. 48, n 173, 1996, p. 30-48.

Besançon Alain, La falsification du bien (Soloviev et Orwell), Paris, Julliard, $1985,222 \mathrm{p}$.

Chtcherbinin Mikhail, «L'Occident et l'Orient, Friedrich Nietzsche et Vladimir Soloviev", Essais sur le discours de l'Europe éclatée [Grenoble], n 13, 1997, p. 97-105.

Clément Olivier, «Vladimir Soloviev, théologien de la modernité ? , Contacts, vol. $47, n^{\circ} 169,1995$, p. 35-45.

Colloque Vladimir Soloviev (1975) - Nouvelles de l'Institut catholique de Paris, $\mathrm{n}^{\circ}$ 1, mai 1979, $160 \mathrm{p}$.

Dennes Maryse, «Identité et appartenance religieuse: l'apport de Vladimir Soloviev», Bulletin de littérature ecclésiastique, 97, 1996, p. 375-391.

Guettée Wladimir, La Russie et son Église, lettre à M. V. Soloviev à propos de sa brochure intitulée "l'Idée russe”, Paris, Fischbacher et Bruxelles, veuve Monnom, 1888, $33 \mathrm{p}$.

— Paris, Fraternité orthodoxe Saint Grégoire Palamas, 1990, 135 p.

Herbigny Michel d', Un Newman russe: Vladimir Soloviev (1853-190o), Paris, Beauchesne (Bibliothèque Slave), 1911, 336 p.

Herman Maxime, "Le sens de la guerre d'après Vladimir Soloviev", Revue des études slaves, XXXVIII, 1961, p. 99-104.

Herman Maxime, Vie et cuvre de Vl. Soloviev, Fribourg, Éditions universitaires, 1995, 157 p. [introduction à Vladimir Soloviev, Crise de la philosophie occidentale, Paris, Aubier-Montaigne (Bibliothèque philosophique), 1947].

Hessen Serge, «La lutte entre l'utopie et l'autonomie du bien dans la philosophie de Dostoïevski et de Vl. Soloviev", Le monde slave, 1930: $\mathrm{n}^{\circ}$ 2, p. 224-250; $n^{\circ} 4$, p. 41-58; no 7 , p. 18-38; no 8, p. 186-201. 
Kojevnikov Alexandre [Kojève], «La métaphysique religieuse de Vl. Soloviev», Revue d'histoire et de philosophie religieuse, $\mathrm{n}^{\circ} 14,1934, \mathrm{p} .534-554 ; \mathrm{n}^{\circ} 15$, 1935, p. 110-152.

LALoy Jean, «Esprit et Histoire. À propos de Vladimir Soloviev», Les quatre fleuves, 9, 1979, p. 125-133.

LAUbier Patrick de et al., Vladimir Soloviev, Jacques Maritain et le personnalisme chrétien, Paris, Parole et Silence, 2008, 196 p.

Lossky Nicolas, Histoire de la philosophie russe des origines à 1950, Paris, Payot, 1954, p. 82-134.

LubAc Henri de, La postérité spirituelle de Joachim de Flore, t. II, Paris, Lethielleux, 1981, p. 406-434.

Mүкоцаiтis Vincas, L'esthétique de Vladimir Soloviev [thèse], Würzburg, Druck von C. J. Becker, 1923, 77 p.

Porret Eugène, La philosophie chrétienne en Russie, Neuchâtel, La Baconnière, 1944, $175 \mathrm{p}$.

Poustooutof Jean, «La justification ecclésiologique de l'idée de "nation chrétienne" et la coexistence de différentes Églises dans un même lieu », Irenikon, vol. $65, \mathrm{n}^{\circ} 3,1992$, p. 387-399.

Rouleau François, "Soloviev (Vladimir)», in Dictionnaire de spiritualité ascétique et mystique, André Derville, Paul Lamarche, Aimé Solignac (dir.), Paris, Beauchesne, 1989, vol. 14, p. 1024-1033.

Rupp Mgr Jean, Un levier pour l'œecuménisme: Wladimir Solowiew, Bruxelles, Vie avec Dieu, 1975, 132 p.

Sigov Konstantin, «Vladimir Soloviev, penseur du Bien», Istina, vol. 51, nº 2, 2006, p. 164-170.

Société D’études philosophiques et Religieuses Vladimir Soloviev, Ecuménisme et eschatologie selon Soloviev, Paris, F.-X. de Guibert (Sagesse chrétienne), 1994, $182 \mathrm{p}$.

Sokologorsky Igor, «Platon et le tournant des dernières années de Soloviev», Le temps philosophique [université Parix X-Nanterre], $\mathrm{n}^{\circ}$ 7, 2000, Présences grecques dans la pensée russe, Annick Charles-Saget (dir.), p. 87-97.

Solowiew Serge M., Vie de Wladimir Solowiew par son neveu, Paris, Éditions S.O.S., 1982 (préface, notes et traduction de $\mathrm{M}^{\mathrm{gr}}$ Jean Rupp), $473 \mathrm{p}$. 
Strémooukhoff Dimitri, Vladimir Soloviev et son œuvre messianique, Lausanne, l'Âge d'homme, s.d. [1975], 352 p.

Tenace Michelina, La beauté unité spirituelle dans les écrits esthétiques de Vladimir Soloviev, Troyes, Fates, 1993 (préface d'Olivier Clément), 203 p.

Tenace Michelina, «Vladimir Soloviev (1853-190o) un exemple de la fécondité en théologie de la philosophie religieuse russe», Transversalités, 89, 2004, p. 113-129.

Toinet Paul, Vladimir Soloviev, chevalier de la Sophia, Genève, Ad Solem, 2001, $236 \mathrm{p}$.

Vladimir Soloviev, introduction et choix de textes traduits pour la première fois par Jean-Baptiste Séverac, Paris, L. Michaud, 1910, 222 p.

Vladimir Solov'ev, la "mutabilité" d'une pensée - Cahiers du monde russe, 42 / 1, 2001: Bernard Dupuy, «S. interprète de Schelling», p. 159-184; François Rouleau, «S. slavophile ou occidentaliste», p. 169-174; Georges Nivat, «S. européen», p. 175-184 (http://monderusse.revues.org/index1169.html).

YAcoub Joseph, «La réconciliation entre Orient et Occident selon Vladimir Soloviev», Istina, vol. 49, n 3, 2004, p. 255-272.

Zenkovsky Basile, Histoire de la philosophie russe, Paris, Gallimard, t. II, 1955 (trad. par Constantin Andronikof), p. 9-73. 